\title{
Treatment of Congenital Divided Nevus of the Eyelid with Excision and Blepharoplasty
}

Soo A Lim, An Young Ryu, Dong Lark Lee, Yong Il Yoon

Department of Plastic and Reconstructive Surgery, Hanil General Hospital, Seoul, Korea

Correspondence: Soo A Lim

Department of Plastic and Reconstructive Surgery, Hanil General Hospital 308 Uicheon-ro, Dobong-gu, Seoul 132-703, Korea

Tel: +82-2-901-3109, Fax: +82-2-901-3104

E-mail:zzcrunch@naver.com

No potential conflict of interest relevant to this article was reported.

Received: 26 Dec 2011 • Revised: 16 Mar 2012 • Accepted: 26 Mar 2012 pISSN: 2234-6163 • elSSN: 2234-6171

http://dx.doi.org/10.5999/aps.2012.39.4.437 • Arch Plast Surg 2012;39:437-439

Copyright (C) 2012 The Korean Society of Plastic and Reconstructive Surgeons

This is an Open Access article distributed under the terms of the Creative Commons

Attribution Non-Commercial License (http://creativecommons.org/licenses/by-nc/3.0/)

which permits unrestricted non-commercial use, distribution, and reproduction in any

medium, provided the original work is properly cited.

A congenital divided melanocytic nevus in the eyelid is a rare form of congenital melanocytic nevus that occurs in the upper and lower eyelids of one eye. As the lesion also exists in the area where the edge of the upper eyelid meets the edge of the lower eyelid, the nevus characteristically appears as a single large nevus when the patient closes the affected eye [1]. Although a congenital facial nevus rarely becomes malignant, it may cause psychological stress during the childhood and the nevus in the eyelids may cause functional problems. Thus, if possible, the nevus should be excised and the defect site should be reconstructed. We report a rare case of a congenital divided melanocytic nevus in the eyelids that was treated successfully with excision and blepharoplasty.

A 14-year-old boy who was referred from the Department of Dermatology had a congenital dark brown plaque lesion in the upper and lower eyelids of his left eye. The dark brown plaques sized $1 \times 1 \mathrm{~cm}$ with uneven surfaces were observed in the upper and lower eyelids of his left eye (Fig. 1). According to the classification of periorbital nevus by area as described by Margulis et al. [2] the lesions belonged to zone 2 . As it was concluded that the lesions could be reconstructed without a skin graft or flap surgery, excision of the nevus and blepharoplasty were performed under local anesthesia. Because there was dermatochalasis in the upper eyelid, a design for doubleeyelid formation was drawn. The inferior incision line 
was marked at $5 \mathrm{~mm}$ from the lash and the upper line was drawn at the margin of the nevus. After this, the entire upper eyelid, including the nevus, was excised, and a 5-mm-wide strip of orbicularis muscle and fat were removed (Fig. 2). At 3 points, the tarsal plate and the skin of the eyelid were sutured using 6-0 Nylon to form a double-eyelid line, and then the skin flap was continuously sutured using a 7-0 black silk suture. To achieve symmetry for both eyes, blepharoplasty of the unaffected eye was also performed. To remove the nevus in the lower eyelid and reconstruct the lower eyelid, a design for a blepharoplasty of the lower eyelid, including the nevus, was drawn, and then a full-thickness excision was performed. As there was a concern about the possibility of the sclera showing due to the large volume of the excised skin flap, the anterior part of the eyelid of the orbicularis oculi muscle was preserved. The lateral area of the anterior part of the eyelid of the orbicularis oculi muscle was anchored to the periosteum at the lateral orbital tubercle with interrupted sutures using 5-0 Vicryl to provide additional support to the lower eyelid (Fig. 3). The removal of the nevus and the

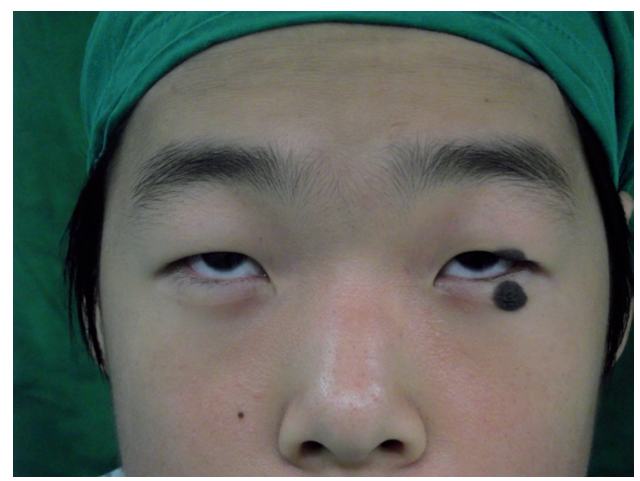

Fig. 1.

Preoperative photograph of congenital divided nevus of the left upper and lower eyelids. When the eyelids are closed the eye appears to be covered by one large nevus. reconstruction took about 1 hour. No particular complications such as post-operative hematoma or a gaping wound were observed. Four days after the surgery, the sutures were removed. Six months later, the mild asymmetry had considerably improved and the patient was very satisfied.

A congenital divided melanocytic nevi in the eyelid characteristically appears as a single nevus because the edges of the upper and lower parts meet each other when the eye is closed [1]. Due to this characteristic, a congenital divided melanocytic nevus is called a 'kissing nevus'. In embryology, the development of the eye starts at 22 days from the onset of pregnancy, when the neural groove of the midbrain thickens and the eyelid is formed at 6-8 weeks of pregnancy. The eyelids remain connected until the 23 rd week of pregnancy [3]. During this period, when the eyelids are connected, melanocytes migrate from the neural groove to the epidermal layer, and through this developmental process, a divided melanocytic nevus can be formed. Margulis et al. [2] classified the zones where a nevus occurs into the following 8 zones, based on the range of the nevus: from the eyebrows

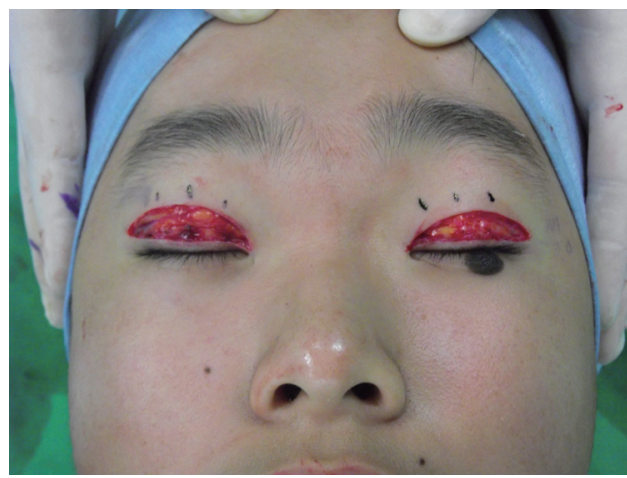

Fig. 2. Resection of the entire nevus and the underlying orbicularis muscle.
Intraoperative photograph and postoperative findings. (A) The nevus of the lower lid was excised

and the lateral border of the muscle flap was anchored to the periosteum at the lateral orbital tubercle. (B) Six months followup result of excision of congenital divided nevus and blepharoplasty.
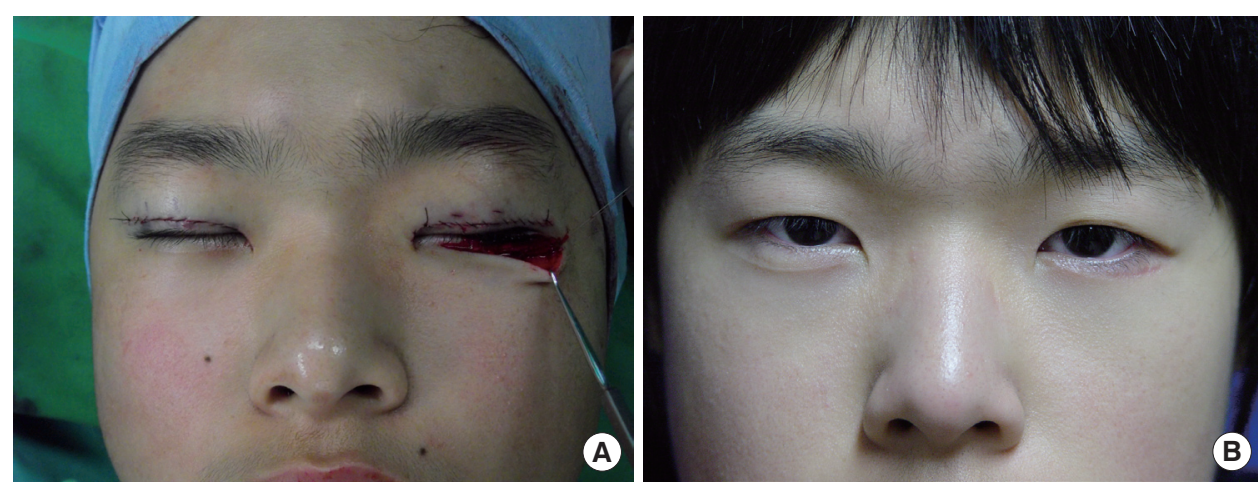
to the upper eyelid wrinkle; from the upper eyelid wrinkle to the upper eyelid borderline; the eyelash area; the lower eyelid boundary to the lower eyelid wrinkle; the isthmus area; the medial area of the eyelid; the forehead area close to the eyebrow; and the nose and canthus.

Most people with congenital melanocytic nevus in the eyelid or the periorbital area have considerable functional and aesthetic stress. In addition, for a palpebra with serious deformation, the suitable timing of surgery is preschool age, as the patient may be teased [4]. The most appropriate type of reconstructive procedure should be selected according to the anatomical units and size. A lesion that is smaller than $1 / 4$ of the upper and lower eyelids can be reconstructed by advancing a nearby skin flap. A lesion that is large can be reconstructed with a full-thickness graft using a retroauricular flap, a lesion in an area that is close to the eyebrow can be reconstructed using an island flap and full-thickness flap surgery, and a large lesion on the forehead and the isthmus area can be reconstructed via local flap surgery using a tissue expander. As the lesions in the patient in this case belonged to zone 2 and caused no malformation of the eyelid, the functional and aesthetic problems could be solved using a relatively simple procedure.

Although no case of a congenital divided melanocytic nevus that became malignant has been reported, it is considered desirable that lesions be excised as completely as possible. Although laser irradiation can be performed at certain intervals, it has the disadvantage of possibly leaving a pigmented lesion after the treatment.

A skin graft after complete excision is commonly performed [2]. However, it may be difficult to have satisfactory results with a skin graft due to the difference in the color and texture of the skin. In this case, the upper eyelid scar was successfully hidden and a satisfactory outcome was achieved via complete anatomical excision of the nevi and via reconstruction using blepharoplasty.

No particular complications were observed in the 6 months follow-up, and the patient's psychological stress due to the nevus was cleared and he was very satisfied (Fig. 3).

\section{References}

1. McDonnell PJ, Mayou BJ. Congenital divided naevus of the eyelids. Br J Ophthalmol 1988;72:198-201.

2. Margulis A, Adler N, Bauer BS. Congenital melanocytic nevi of the eyelids and periorbital region. Plast Reconstr Surg 2009; 124:1273-83.

3. Sevel D. A reappraisal of the development of the eyelids. Eye (Lond) 1988;2:123-9.

4. Bellier-Waast F, Perrot P, Duteille F, et al. Surgical treatment for giant congenital nevi: what are the psychosocial consequences for the child and family? Ann Chir Plast Esthet 2008;53:408-14. 